\title{
8DEstablishment and validation of a hypoxia-related signature predicting prognosis in hepatocellular carcinoma
}

\author{
Congbo Cai ${ }^{1}$, Lei Yang ${ }^{1}$ and Kena Zhou ${ }^{2 *}$
}

\begin{abstract}
Background: Hypoxia plays a crucial role in immunotherapy of hepatocellular carcinoma (HCC) by changing the tumor microenvironment. Until now the association between hypoxia genes and prognosis of HCC remains obscure. We attempt to construct a hypoxia model to predict the prognosis in HCC.

Results: We screened out 3 hypoxia genes (ENO1, UGP2,TPI1) to make the model, which can predict prognosis in HCC. And this model emerges as an independent prognostic factor for HCC. A Nomogram was drawn to evaluate the overall survival in a more accurate way. Furthermore, immune infiltration state and immunosuppressive microenvironment of the tumor were detected in high-risk patients.

Conclusion: We establish and validate a risk prognostic model developed by 3 hypoxia genes, which could effectively evaluate the prognosis of HCC patients. This prognostic model can be used as a guidance for hypoxia modification in HCC patients undergoing immunotherapy.
\end{abstract}

Keywords: Hypoxia, Hepatocellular carcinoma, GSEA, Tumor microenvironment, Risk model, Immune response

\section{Background}

Liver cancer is a common malignant tumor with poor prognosis and high mortality, ranking the fourth in tumor death [1]. HCC accounts for $75-85 \%$ in liver cancer and is the leading cause of death [2]. Although continuous improvement has been made in HCC therapy, the 5-year survival rate of $\mathrm{HCC}$ patients is still less than $20 \%[3,4]$. As is known to all, OS of HCC patients is closely related to the tumor stage. And it is conducive to effective communication between clinicians and patients if the prognosis of individual HCC could be evaluated in an accurate and convenient way.

Tumor growth affects the surrounding microenvironment, and the tumor microenvironment (TME)

\footnotetext{
*Correspondence: kenazhou@126.com

${ }^{2}$ Gastroenterology Department of Ningbo No. 9 Hospital,

Ningbo 315000, Zhejiang, China

Full list of author information is available at the end of the article
}

promotes tumor progression [5]. Substantial data suggest that hypoxia is an important manifestation of TME $[6,7]$. HCC has a high level of vascularization, and lots of oxygen is consumed when tumor grows rapidly. Therefore, TME in HCC seems to be in a statement of low oxygen due to insufficient oxygen supply [8]. Previous study has demonstrated that $\mathrm{HCC}$ is the most serious tumor of hypoxia [9]. Abundant evidence is showing that hypoxia can induce an increase in tumor malignancy, including tumor progression, invasion and metastasis, contributing to poor prognosis in HCC $[10,11]$. Nowadays immune checkpoint inhibitors (ICI) have become an effective immunotherapy and are popularly used in the treatment of HCC [12, 13]. Meanwhile increasing literature indicated that hypoxic microenvironment would decrease the treatment sensitivity in ICI therapy [7]. And improving the hypoxic microenvironment exhibited a more efficient effect on ICI immunotherapy [14]. Therefore, we put forward that hypoxia-related genes could be used 
to predict the prognosis of $\mathrm{HCC}$ and help the choice of hypoxia ameliorating agents during ICI immunotherapy. Currently there is a lack of hypoxia prognostic models in HCC.

In this study, we developed and validated a prognostic model composed of hypoxia-related genes, which can predict the OS of HCC patients. And it is hopefully to be applied as a potential reference for the use of hypoxia ameliorant during immunotherapy in $\mathrm{HCC}$, so as to improve the prognosis in $\mathrm{HCC}$.

\section{Results}

\section{Clinical characteristics of the enrolled participants}

The RNA-Seq expression and corresponding clinical information of $232 \mathrm{HCC}$ patients were downloaded from the International Cancer Genome Consortium (ICGC) database. Analogously, characteristics of 371 HCC patients were obtained from the Cancer Genome Atlas (TCGA) database. Details about the information of training and validation groups are exhibited in Table 1.

\section{Establishment of the hypoxia risk prognostic model}

The genome related to hypoxia was acquired from the gene set enrichment analysis (GSEA) website. These 200 hypoxia-related genes are widely accepted to make research in various clinical studies (See Additional file 1: Data S1 for details). We used the STRING online database (http://string-db.org) to construct a protein-protein interaction network (PPI) for those 200 hypoxia genes (Fig. 1A). The top 50 genes with higher degree

Table 1 Summary clinical characteristic of HCC patients

\begin{tabular}{|c|c|c|}
\hline Characteristics & $\begin{array}{l}\text { Training group } \\
(\text { ICGC } \mathbf{N}=232)\end{array}$ & Test group $($ TCGA $N=371)$ \\
\hline \multicolumn{3}{|l|}{ Age category } \\
\hline$<65 / \geq 65 /$ NA & $83 / 149 / 0$ & $221 / 149 / 1$ \\
\hline \multicolumn{3}{|l|}{ Gender } \\
\hline Male/Female & $171 / 61$ & $250 / 121$ \\
\hline \multicolumn{3}{|l|}{ Vital status } \\
\hline Alive/dead & $189 / 43$ & $241 / 130$ \\
\hline \multicolumn{3}{|l|}{ Grade } \\
\hline G1/G2/G3/G4/NA & NA & $55 / 177 / 122 / 12 / 5$ \\
\hline \multicolumn{3}{|l|}{ Tumor stage } \\
\hline |/II/III/IV/NA & $36 / 106 / 71 / 19 / 0$ & $171 / 86 / 85 / 5 / 24$ \\
\hline \multicolumn{3}{|l|}{ T stage } \\
\hline $\mathrm{T} 1 / \mathrm{T} 2 / \mathrm{T} 3 / \mathrm{T} 4 / \mathrm{NA}$ & NA & $181 / 94 / 80 / 13 / 3$ \\
\hline \multicolumn{3}{|l|}{ M stage } \\
\hline M0/M1/MX & NA & $266 / 4 / 101$ \\
\hline \multicolumn{3}{|l|}{ N stage } \\
\hline N0/N1/NA & NA & $252 / 4 / 115$ \\
\hline
\end{tabular}

NA: Clinical data are unknown of interaction were preliminarily identified to play an important role in the hypoxia process of HCC (Fig. 1B). Univariate cox regression analysis determined that 8 hypoxia-related genes were significantly related to the OS of HCC patients in the ICGC training group $(P<0.001)$ (Fig. 1C). These 8 prognostic-related genes were further analyzed in Least Absolute Shrinkage and Selection operator (LASSO) regression to establish a hypoxic prognosis model for HCC patients. Ultimately, three genes (ENO1, UGP2, TPI1) were selected for the construction of hypoxia prognostic model according to the lamda value in LASSO regression. Among them, ENO1 and TPI1 are risk genes, and UGP2 is a protective gene (Fig. 1D-F). The three prognostic genes in the training and verification groups are closely correlated with each other, positive relation in red and negative in green (Fig. 1G, H).

\section{Validation of the hypoxia prognostic model}

We use the Kaplan-Meier $(\mathrm{K}-\mathrm{M})$ curve and the risk curve to further validate the hypoxia prognosis model. $\mathrm{K}-\mathrm{M}$ curves of ENO1, UGP2 and TPI1 in the ICGC queue were drawn for concise analysis. The survival rate of ENO1 and TPI1 high expression group decreased $(P<0.01)$, suggesting that they are risk genes (Fig. 2A, $C)$. On the contrary, the survival rate of the UGP2 high expression group increased $(P<0.01)$, indicating that UGP2 is a protective gene (Fig. 2B). Likewise, similar results were confirmed in TCGA (Fig. 2D-F).

All specimens are divided into high- and low-risk groups on the basis of the median risk score as a cutoff. The K-M curve in the ICGC cohort showed that the survival rate of patients in the high-risk group was significantly lower than that in low-risk group $(P<0.01)$ (Fig. 3A). And same conclusion was obtained in TCGA validation cohort $(P<0.01)$ (Fig. 3B). Since the risk curve can further verify the reliability of the model, we sorted all patients in the training group in sequence according to risk score (Fig. 3C). We found that the higher risk score is, the shorter survival time a patient has, and the more deaths caused (Fig. 3E). And the expressions of ENO1 and TPI1 increased as the risk score rose, while the expression of UGP2 decreased (Fig. 3G). Definitely we got the same phenomenon in TCGA validation groups (Fig. 3D, F, H). The mortality rate in high-risk group was significantly higher than that in low-risk group (30\% vs. 7\%) (Fig. 3I), which is also verified by external cohort (45\% vs. $23 \%$ ) (Fig. 3J).

\section{Evaluation of the ability of hypoxia prognostic model and the correlation of clinical characteristics}

We performed Receiver operating characteristic (ROC) curves on the data from ICGC and TCGA in order to evaluate the prognostic accuracy of the hypoxia 

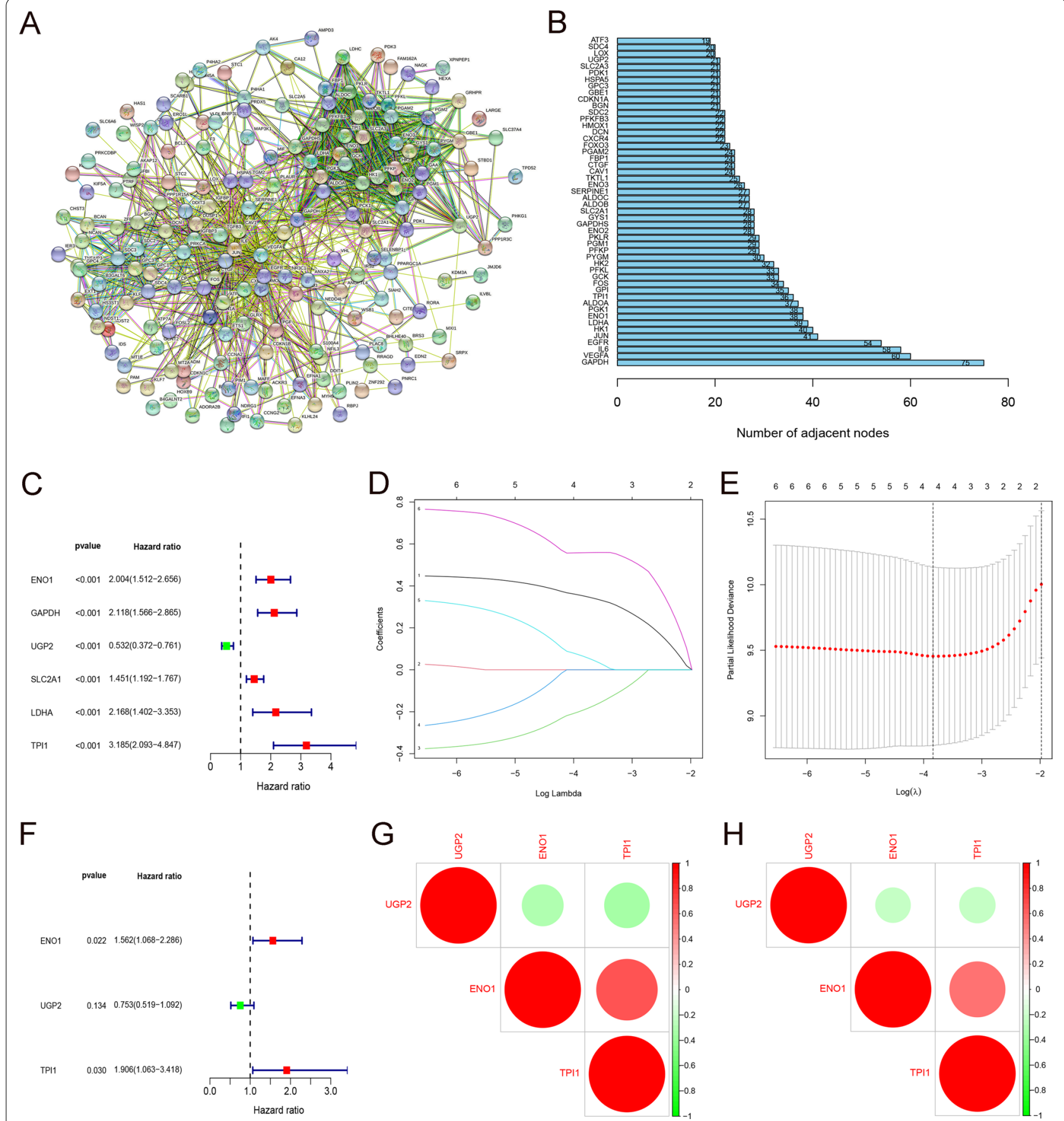

Fig. 1 Prognostic model of hypoxia related genes. A Protein-Protein Interaction network of 200 hypoxia-relevant genes. B Top 50 genes that are most related to each other in PPI network. $\mathbf{C} 6$ hypoxia genes closely related to OS in HCC by univariate cox regression. $\mathbf{D}, \mathbf{E}$ Establishment of 4-gene model via LASSO regression and lamda value. $\mathbf{F}$ Forest maps indicating that ENO1 and TPI1 are risk genes, while UGP2 is a protective gene. G, H Spearman correlation analysis of 3 hypoxia genes in the ICGC and TCGA databases

prognostic model. And value of area under curve (AUC) for 0.5-, 1-, 3-, and 4-year OS in ICGC were 0.727, 0.763, 0.787 , and 0.777 respectively (Fig. 4A). Likewise, the 0.5-, 1-, 3-, and 5-year AUC values in the verification cohort were $0.729,0.724,0.666$, and 0.692 respectively (Fig. 4B). The concordance index (C-index) value of the model in the experimental group is 0.7551 (95\% CI $68.0-83.0 \%$, $P<2.0674 \mathrm{E}-11)$. The $\mathrm{C}$-index value in the verification 

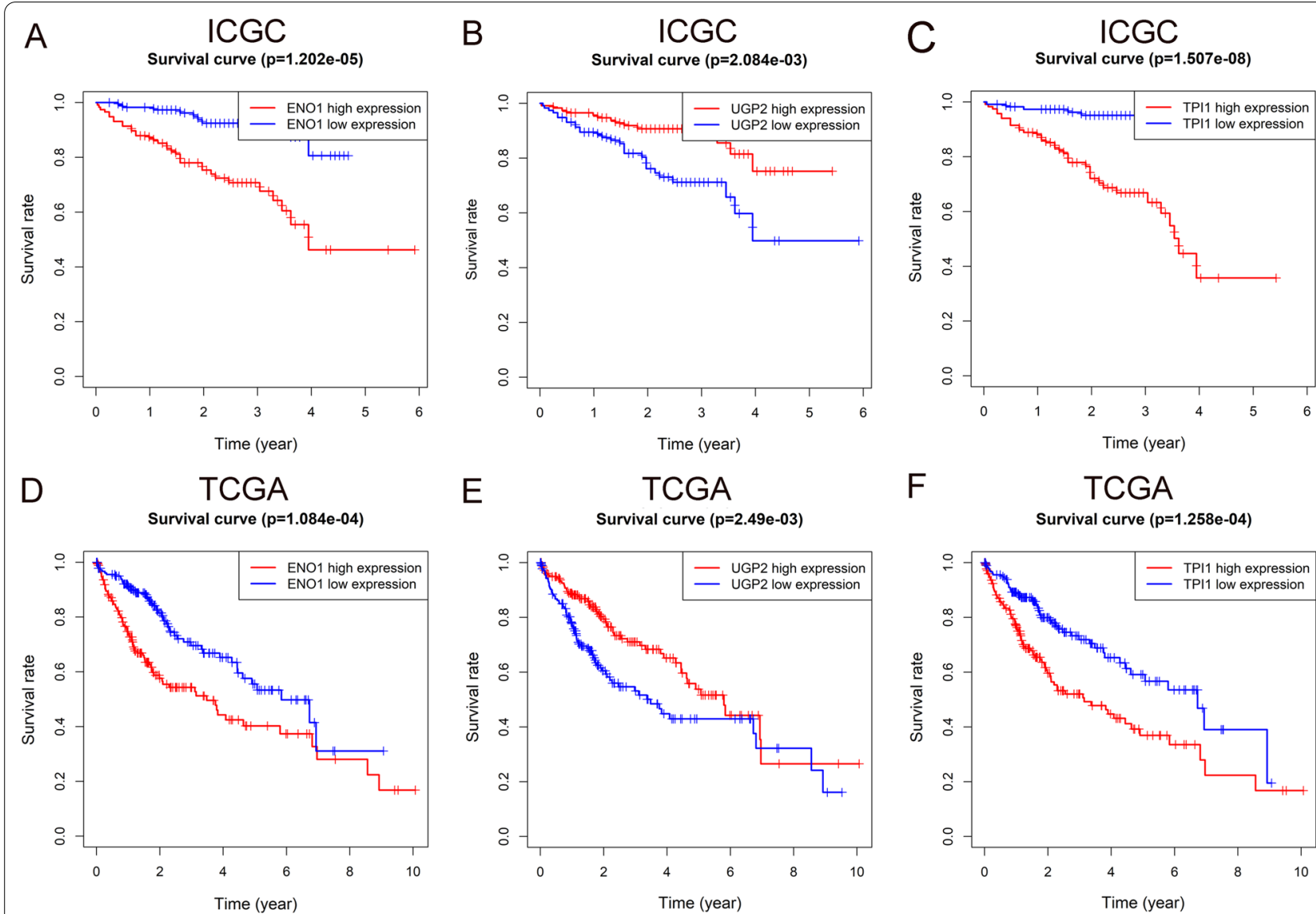

$\mathrm{F}$
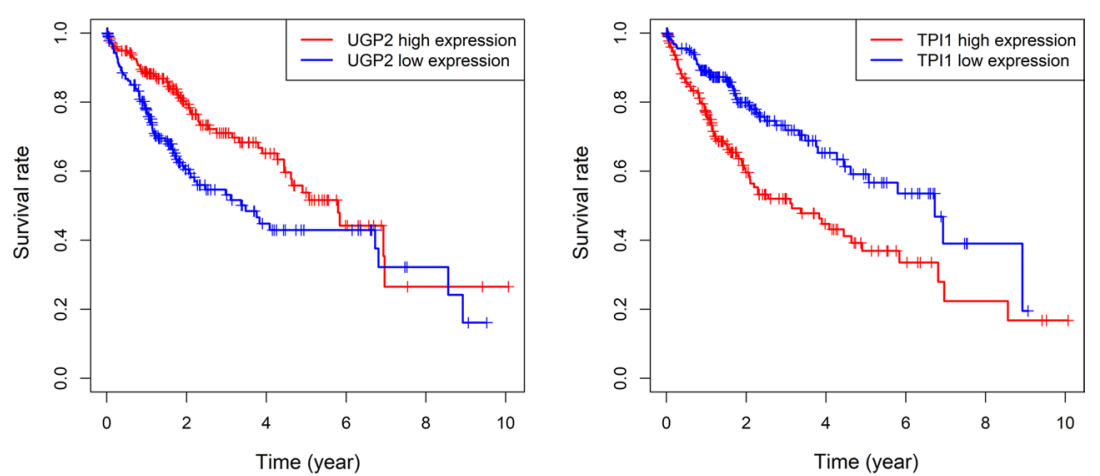

Fig. 2 Survival analysis of hypoxia genes. A-C K-M curves for patients' survival of ENO1, UGP2 and TPI1 in ICGC. D-F K-M curves for patients' survival of ENO1, UGP2 and TPI1 in TCGA

(See figure on next page.)

Fig. 3 Prognostic value and external validation of hypoxia genes. A K-M curves of patients in high- (red) and low-risk groups (blue) of ICGC. B K-M curves of patients in high- (red) and low-risk groups (blue) of TCGA. C, D Sorting patients according to risk score in ICGC and TCGA respectively. E, F Association between survival time and risk score in ICGC and TCGA separately. G, H Relationship between risk score and the expression of ENO1, TPI1 and UGP2. I, J The mortality rate in high- and low-risk groups of ICGC and TCGA

group is $0.6802(95 \%$ CI $63.1-73.0 \%, P<8.6844 \mathrm{E}-13)$. The $P$ value of the model in the experimental group and the verification group were both $<0.01$, indicating that the model has a strong predictive ability. Both the training and validation groups confirmed the good predictive ability of the established prognostic model.

Stage is a traditional clinical tumor stratification with strong practicability in daily clinic. We focused on the relationship between three hypoxia genes and Stage. A heat map was created to explain the association between gene expression level and stage stratification. The expression of ENO1 and TPI1 increased in higher stage levels $(P<0.01)$, while UGP2 expression was weakened $(P<0.01)$ (Fig. 4 C).
Moreover, quantity analysis was made to identify the relationship between the three hypoxic prognostic genes and the stage level. As the stage level goes up, the expressions of ENO1 and TPI1 increase, and the expression of UGP2 decreases $(P<0.05)$ (Fig. 4E). The expression of ENO1 in the validation group was the same as that in the experimental group. And the expression of UGP2 and TPI1 has no obvious relationship with stage (Fig. 4D, F).

\section{The independent role of hypoxia prognostic model and the predictive nomogram}

We also assessed whether the hypoxia prognostic model is independent from other traditional clinical 


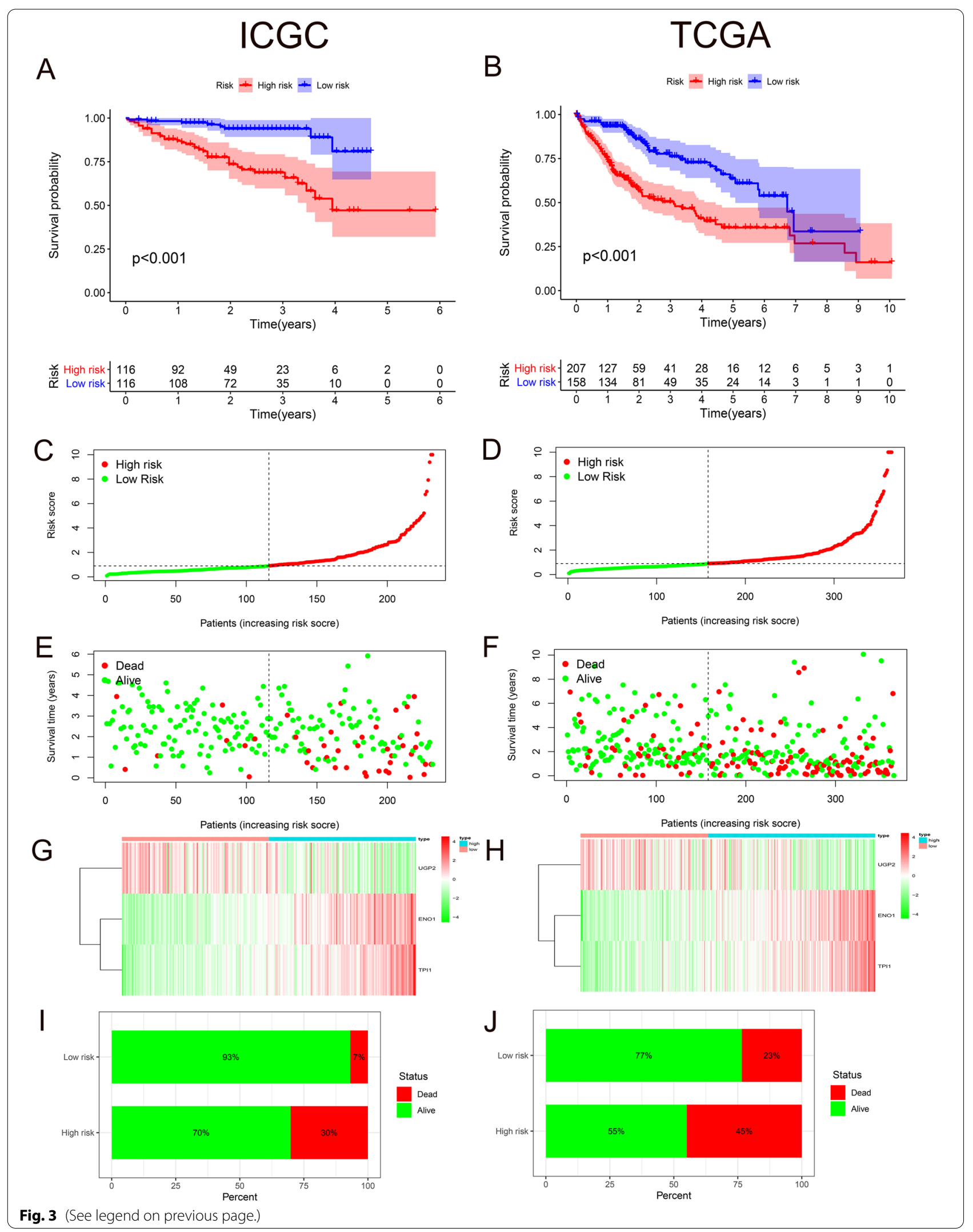




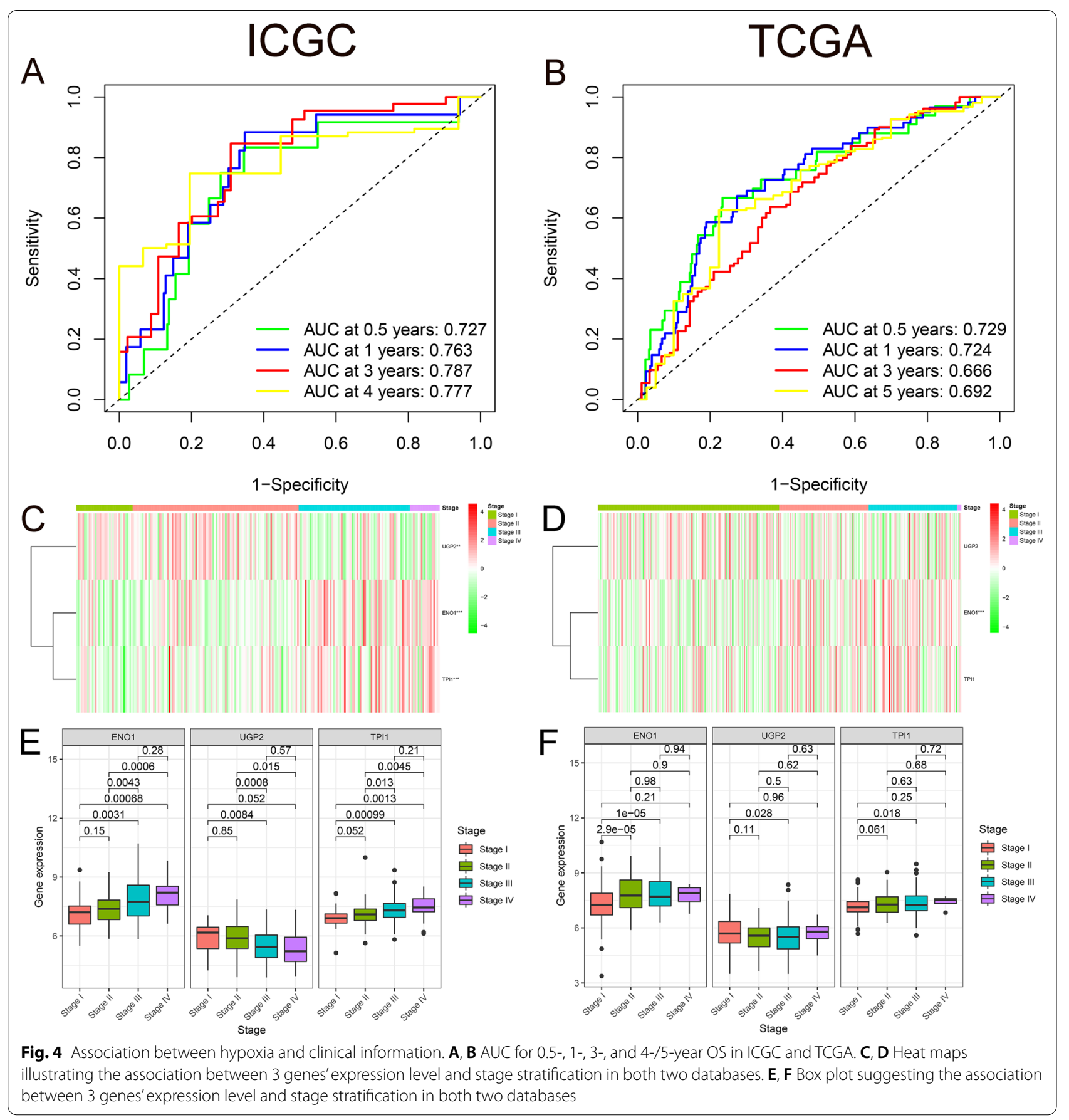

features. The results showed that stage stratification and risk score were independent prognostic factors of OS in the ICGC cohort, both with $P$ values $<0.01$ (Fig. 5A, C). And the same finding is suggested in TCGA validation group (Fig. 5B, D). In addition, we produced a nomogram for $\mathrm{HCC}$ patients, which can be used to quantitatively assess the survival time of each individual. The contents included in the nomogram are clinical features such as gender, age, stage, as well as risk score. Every item has a specific score, and the final score is summed up of all factors. A vertical line through the integrated score corresponds to the survival probabilities of 1-, 3-, and 5-years (Fig. 5E). 


\section{ICGC}

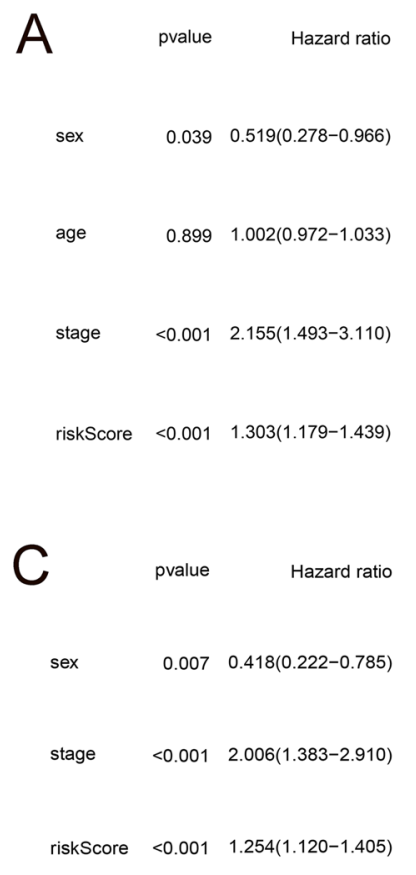

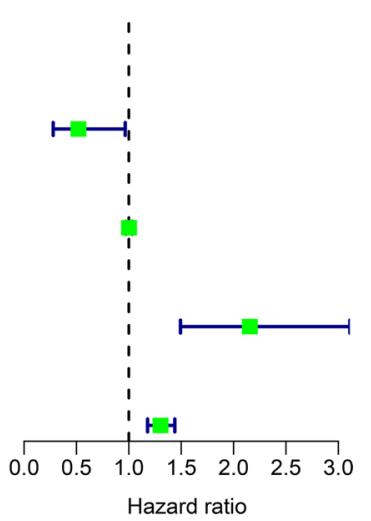

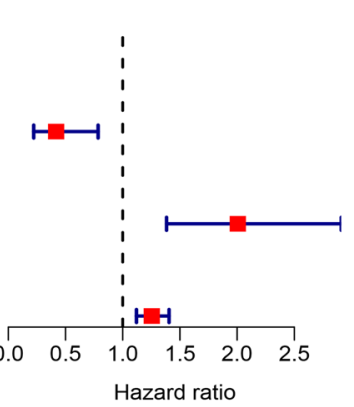

\section{TCGA}

B

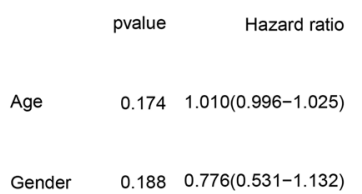

Gender $\quad 0.188 \quad 0.776(0.531-1.132)$

Grade $\quad 0.330 \quad 1.133(0.881-1.457)$

Stage $\quad<0.001 \quad 1.680(1.369-2.062)$

riskScore $\quad<0.001 \quad 1.125(1.054-1.200)$

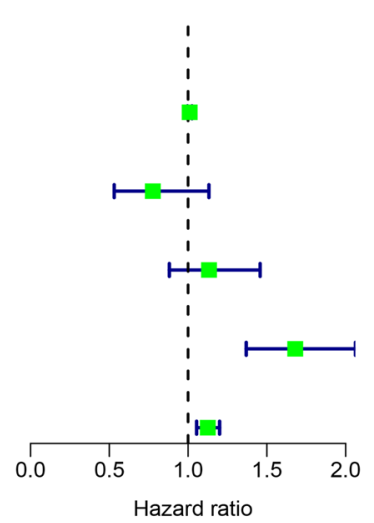

D

pvalue Hazard ratio

Stage $\quad<0.001 \quad 1.605(1.297-1.985)$

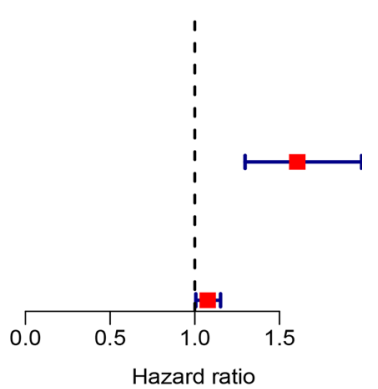

riskScore $\quad 0.033 \quad 1.076(1.006-1.152)$

$E$

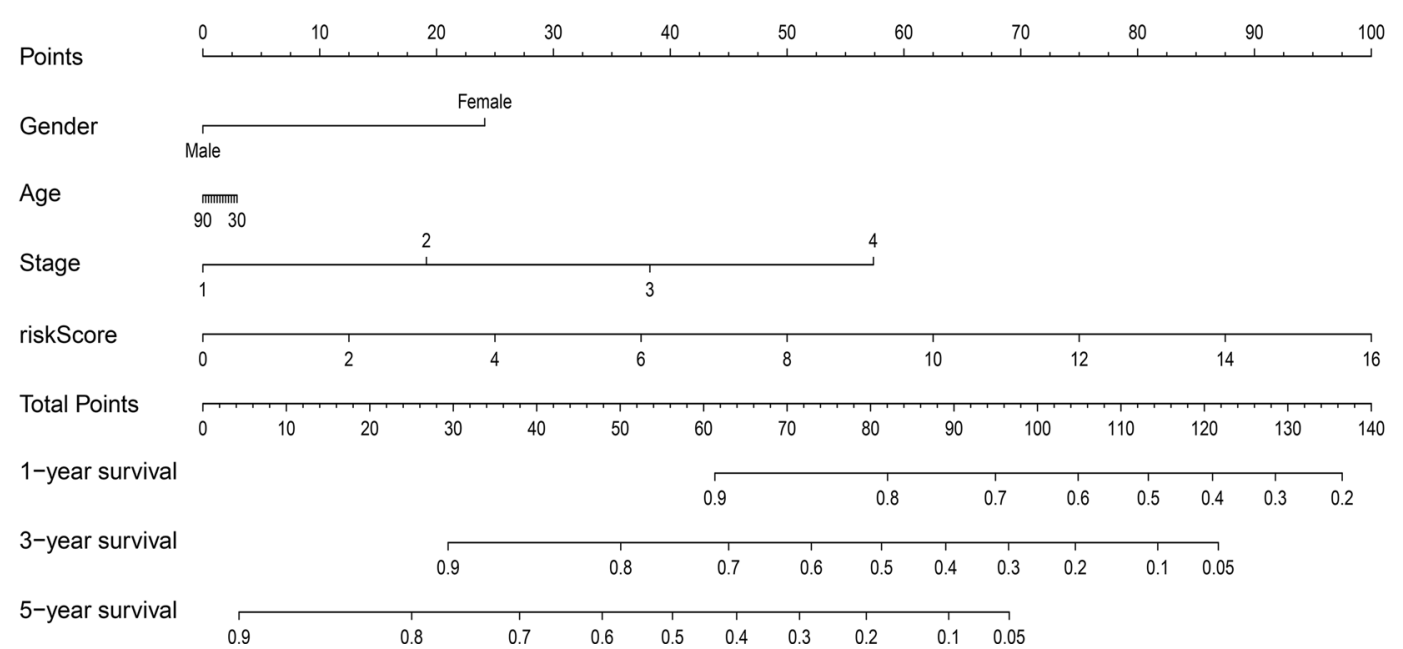

Fig. 5 Prognostic value of hypoxia related genes and nomogram. A-D Independence tests between risk score and clinical characteristics both in ICGC and TCGA. E A nomogram predicting the OS in HCC

\section{Functional analysis of the prognostic model}

For the purpose of investigating the possible signal pathways related to hypoxia in the progression of HCC, we use the GSEA method to compare the pathway differences between people in high- and lowhypoxia situations. GSEA results told us that DNA
Repair, Glycolysis, and Unfolded Protein responses were enriched in both ICGC and TCGA cohorts (Fig. 6A-F). 


\section{ICGC}

A Enrichment plot: HALLMARK_DNA_REPAIR

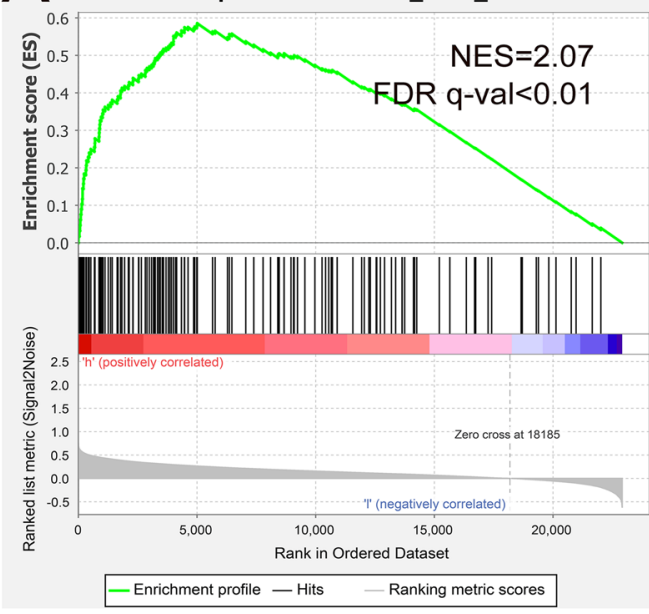

Enrichment plot: HALLMARK_GLYCOLYSIS
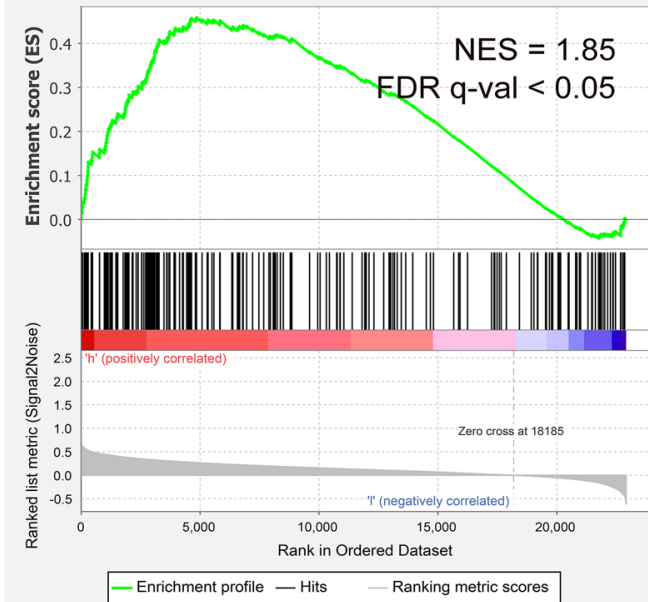

$E$
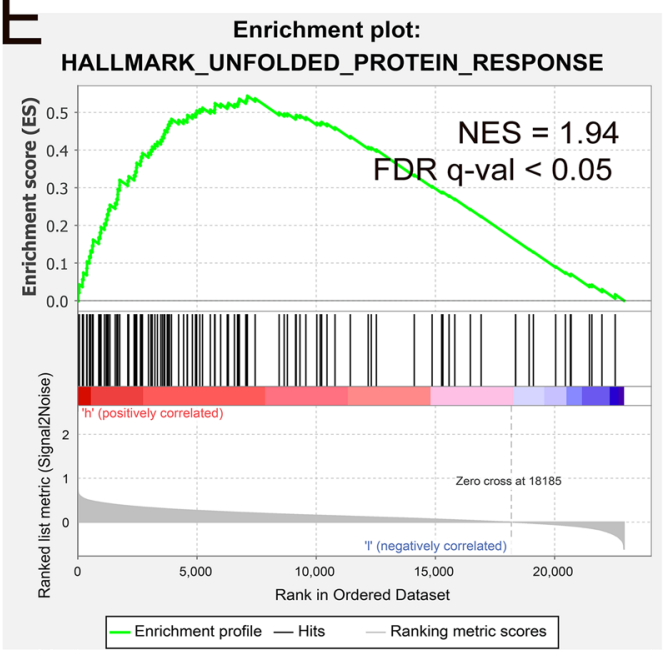

TCGA

B Enrichment plot: HALLMARK_DNA_REPAIR

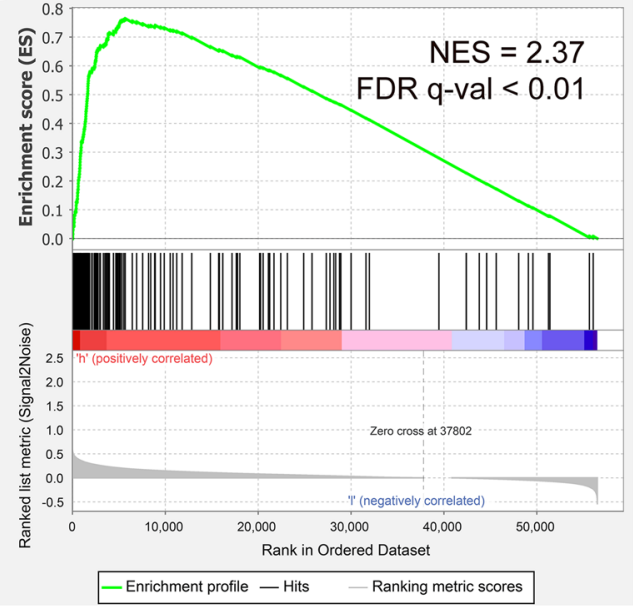

D Enrichment plot: HALLMARK_GLYCOLYSIS

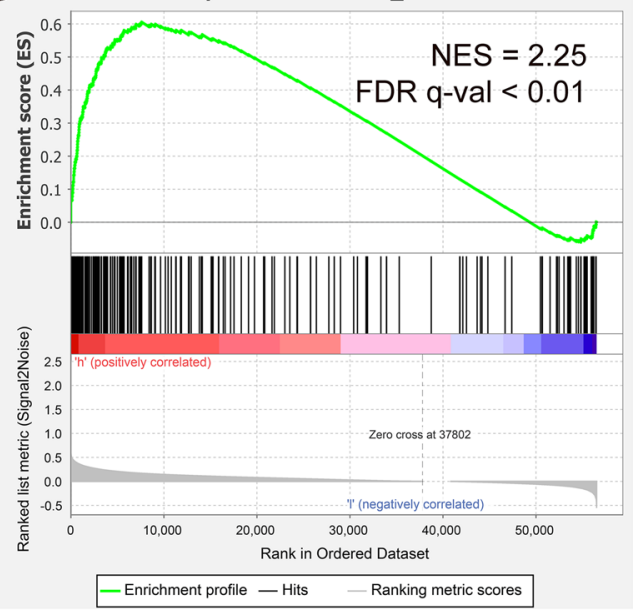

F

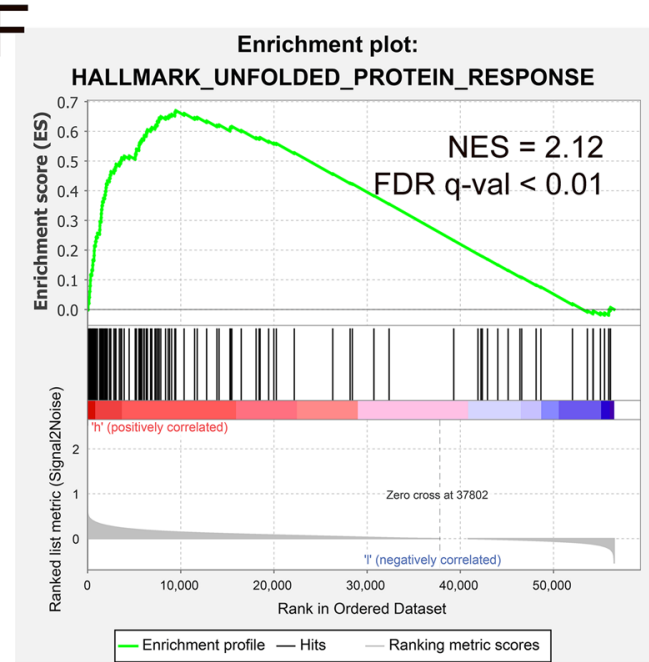

Fig. 6 GSEA enrichment of high- and low-risk groups. A, C, E DNA Repair, Glycolysis, and Unfolded Protein responses were enriched in ICGC. B, D, F DNA Repair, Glycolysis, and Unfolded Protein responses were enriched in TCGA 


\section{Immune cell infiltration among HCC patients in different risk groups}

We used the CIBERSORT algorithm to evaluate the degree of immune infiltration of 22 immune cells in the high- and the low-risk groups. A conclusion of ICGC data was drawn in Fig. 7A, and TCGA in Fig. 7B. B cells naive is suppressed in patients with higher levels of hypoxia (Fig. 7C). And the verification results are shown in Fig. 7D. However, the expression of $\mathrm{T}$ cells is increased in patients with higher levels of hypoxia (Fig. 7E). And the verification group obtained the same phenomenon (Fig. 7F).

\section{The immunosuppressive microenvironments and key immune checkpoints in different risk groups}

Extensive evidence proves that immunotherapy can improve the prognosis of HCC patients significantly [15]. And previous studies suggest that immunosuppressive cytokines play an important role in the TME, especially in the process of tumor development and metastasis [16]. Hence we aim to detect the expression of immunosuppressive genes in high- and low-risk groups of HCC. All immune-related genes were downloaded from the Tracking Tumor Immuno-phenotype website (http://biocc. hrbmu.edu.cn/TIP/index.jsp) (more details in Additional file 2: Data S2). In ICGC cohort, we made a heat map to depict negative immune regulatory genes with differential expression levels $(P<0.05)$ (Fig. 8A). Same graph was produced in TCGA $(P<0.05)$ (Fig. $8 B)$. This demonstrated that many immunosuppressive genes are activated during HCC hypoxia process.

Besides, popular immune checkpoint genes in HCC were also assessed. Our results showed that CTLA-4 and TIM-3 were positively correlated with hypoxia risk score in both ICGC and TCGA $(P<0.01)$ (Fig. 8C, G). And box plots illustrated that CTLA-4 and TIM- 3 are up-regulated in the high-risk group $(P<0.05)$ (Fig. 8D, $\mathrm{H})$. However, the immune checkpoint LAG-3 was negatively correlated with the hypoxia risk score in the ICGC cohort, and positively correlated with the hypoxia risk score in the TCGA cohort $(P<0.01)($ Fig. $8 \mathrm{E})$. Of course same results in box plots of LAG-3 $(P<0.05)$ (Fig. 8F).

\section{Discussion}

The homeostasis of microenvironment is the basis for the stable growth of normal tissues and cells. Predecessors have put forward that the rapid growth of HCC causes a hypoxic TME [17]. Collaboratively, hypoxic TME could promote the deterioration of $\mathrm{HCC}$ and increases aggressiveness [18]. Simultaneously, hypoxia can also change the immune status, affecting the prognosis of HCC. Up to now, there is no suitable prognostic model for HCC hypoxia.

In this study, we found that hypoxia has an important effect on the prognosis and immunotherapy of HCC. We developed and verified a model consisted of 3 hypoxia genes (ENO1, UGP2, TPI1), with good prognostic predictive value. Moreover, the nomogram we drew could
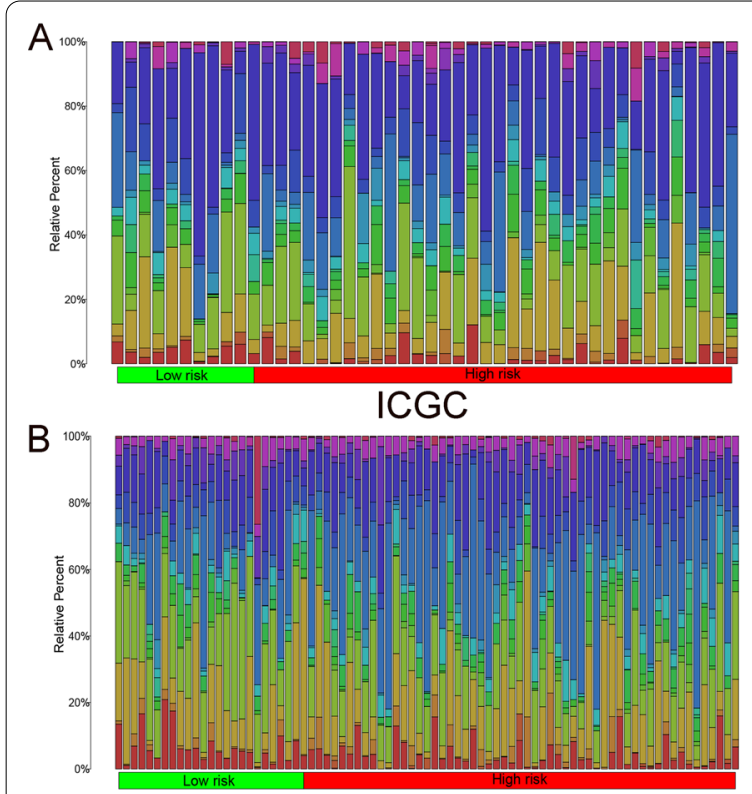

TCGA
C
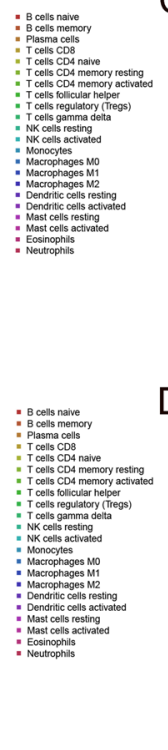

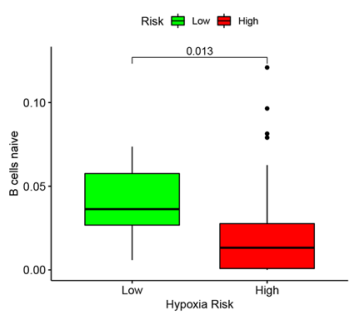

ICGC B cells naive

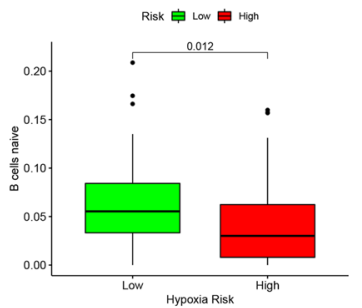

TCGA B cells naive
$\mathrm{E}$

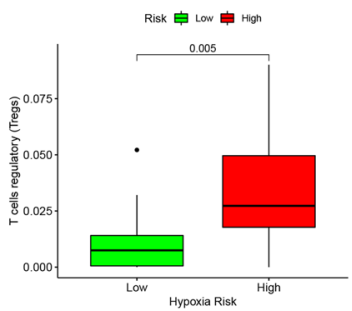

F
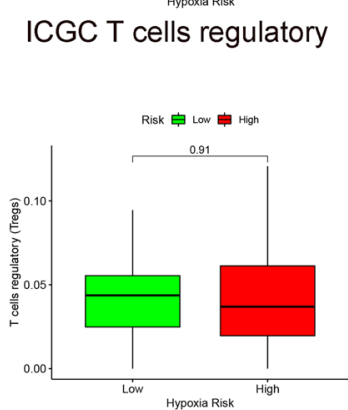

TCGA T cells regulatory

Fig. 7 Immune situation in high- and low-risk groups. A, B Infiltration of 22 immune cells in high-and the low-risk groups of ICGC and TCGA. C, D B cells naïve in high- and the low-risk groups of ICGC and TCGA. E, F T cells regulatory in high- and the low-risk groups of ICGC and TCGA 


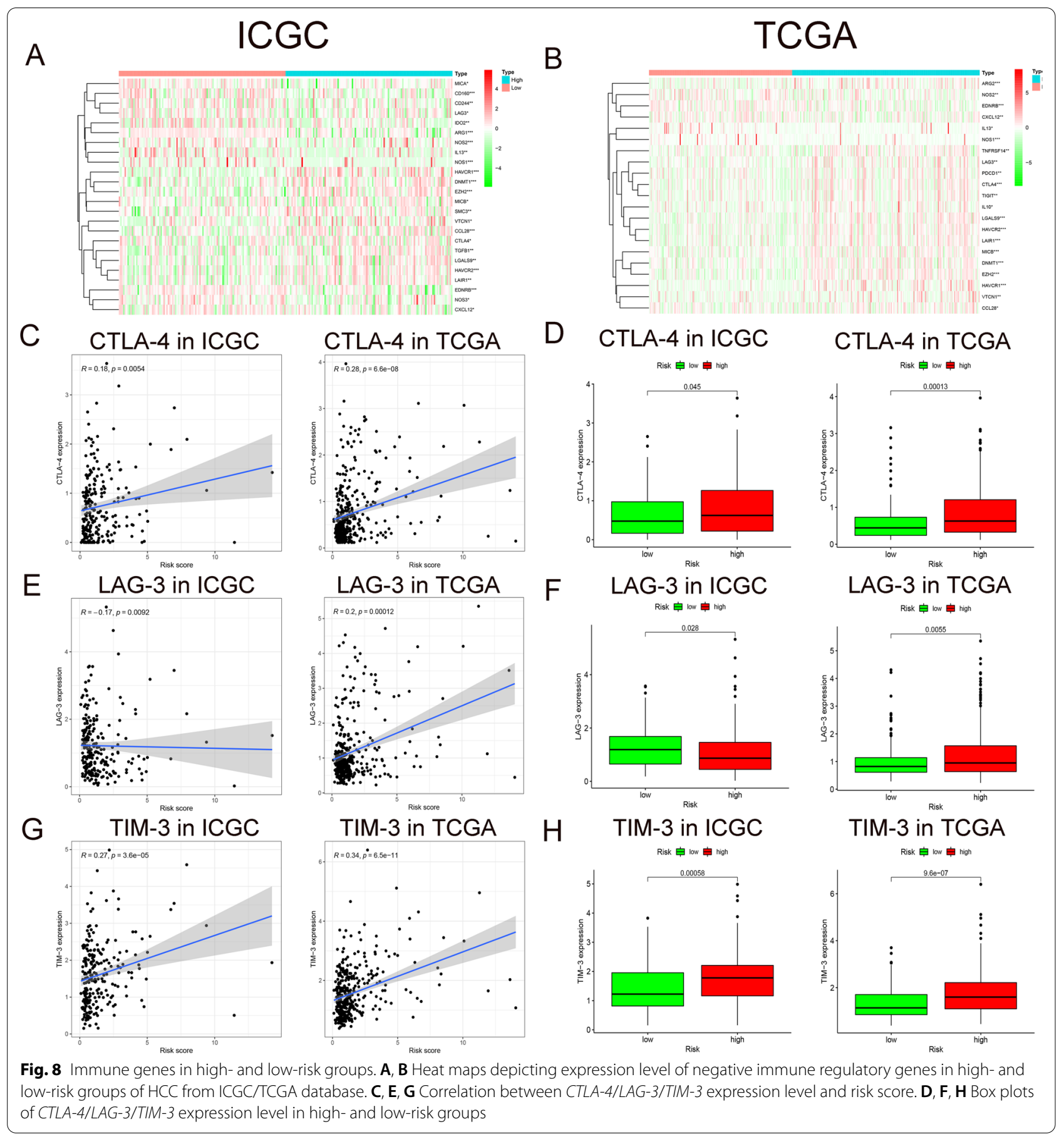

accurately assess the OS of individual $\mathrm{HCC}$ patient, with easier accessibility. And we also found that the hypoxia genes of this model are mainly related to DNA Repair, Glycolysis, and Unfolded Protein response after GSEA enrichment analysis. In addition, we found that the hypoxic TME has an impact on HCC immune infiltration and immune regulation. These discoveries provide a novel insight for predicting the prognosis of HCC patients according to the characteristics of the hypoxic TME. Furthermore, the degree of hypoxia in the TME can be used as a reference indicator for the use of hypoxia ameliorating agents in immunotherapy.

Actually the importance of these hypoxia-related genes has been previously reported in a variety of studies. ENO1 is a key regulator enzyme of glycolysis. Studies have pointed out that the serum ENO1 level in HCC 
patients was significantly higher than that in normal series [19]. Further studies have shown that the up-regulation of ENO1 promoted cell proliferation, migration and invasion of HCC cells [20]. This is consistent with our research. UGP2 is an essential enzyme for glycogen synthesis. Studies have suggested that UGP2 expression was significantly down-regulated in HCC tissues. And down-regulation of UGP2 expression was equal to poor prognosis in HCC patients [21]. Our research demonstrated that UGP2 is a protective gene of HCC.TPI1 is a key enzyme in the process of sugar metabolism and gluconeogenesis. Previous literature has shown that the enhanced expression of TPI1 in HCC cells in vitro inhibits cell growth, migration and invasion. The survival curve shows that the survival rate of patients is worse when TPI1 is in low expression [22]. However, there are also evidence supporting that OS of HCC patients increased with inactivation of the eight hypoxia genes including TPI1 [23]. And our $\mathrm{K}-\mathrm{M}$ curve shows that the survival rate of HCC patients increases when TPI1 is low expressed. We believe that these inconsistent results may be due to different environments in vitro and in vivo. Definitely, this requires more basic research to further clarify the function of TPI1 in HCC patients.

GSEA enrichment analysis can effectively explore the possible biological mechanisms of hypoxia prognostic models. Our results indicate that DNA Repair, Glycolysis and Unfolded Protein response are enriched in high-risk group. Substantial evidence showed that hypoxia could cause genome instability and inhibit the ability of DNA damage repair pathways [24-26]. Glycolysis is the main energy source for HCC in hypoxic environment [27, 28]. Cancer cells are often exposed to hypoxia, nutritional deficiencies, oxidative stress and other metabolic disorders, leading to the activation of endoplasmic reticulum stress and Unfolded Protein response [29-31]. Our results indicate that the above pathways are enriched in high-risk patients, revealing that hypoxia is of vital importance in the occurrence and development of HCC.

Tumors can protect themselves from attack by stimulating immune checkpoint targets, such as PD-1, PD-L1, CTLA-4, LAG-3, and TIM-3 [32-35]. Previous literature showed that PD-1/PD-L1 and CTLA-4 are up-regulated at the transcription level under hypoxic conditions, enhancing the immune escape of cancer cells [36-38]. Here we found that the expression of CTLA- 4 and TIM-3 in hypoxic patients increased, indicating that the hypoxic TME would promote the immune escape of cancer cells. Many studies have suggested that increasing oxygen in the TME could improve the anti-tumor efficacy of PD-1/ PD-L1 and CTLA-4 antibodies [36, 39, 40]. The combination of ICIs and hypoxia ameliorating agents even showed a more effective treatment for HCC patients
[41-44]. Thus it is conceivable that the hypoxia risk score has a latent promising value in the use of hypoxia ameliorant during immunotherapy.

In other words, this study has some limitations. First of all, the process of adjusting the weights of regression coefficients in LASSO might ignore some factors that contribute to the prognosis of HCC. Secondly, different standards of inclusion do exist when collecting information retrospectively in different public databases. For example, in stage stratification, ICGC uses the LCSGJ standard, while TCGA uses the AJCC standard. Finally, the complex interaction between tumor cells and immune cells in the hypoxic microenvironment remains to be further explored. More specific experiments are still needed to verify these findings.

\section{Conclusions}

In short, we establish and validated a risk prognostic model developed by 3 hypoxia genes, which could effectively evaluate the prognosis of HCC patients. This prognostic model can be used as a guidance for hypoxia modification in HCC patients undergoing immunotherapy. It will be of great significance to the manipulation of hypoxic stress in comprehensive and innovative immunotherapy in cancer in the near future.

\section{Methods \\ Datasets}

The training cohort comes from the ICGC database (https://icgc.org/). The validation cohort is from TCGA database (https://portal.gdc.cancer.gov). Both the ICGC and TCGA databases are freely available to the public, and there is no need for institutional ethics committee approval and informed consent. This research strictly follows the acquisition policy and publication guidelines.

\section{Constitution of a risk model}

Hypoxia-related genes are obtained from the GSEA website (https://www.gsea-msigdb.org/). The 200 hypoxia genes in this group are widely recognized by the academic community for the analysis of tumor hypoxia. The PPI pattern is analyzed in the STRING database (https:// www.string-db.org/). Univariate Cox regression analyses was performed to screen out hypoxia genes related to OS $(P<0.001)$. LASSO regression can avoid over-fitting, further optimize the genes selected after univariate cox regression, and delete highly related genes [45]. Finally, multivariate COX regression analysis was carried out step by step to set up a prognostic model. The risk score of HCC patients can be calculated by the following formula: Riskscore $=\sum_{i=1}^{N}(\mathrm{Ei} * \mathrm{Ci})$, while Ei was the expression value of every three hypoxia genes, and 
$\mathrm{Ci}$ was the corresponding multivariable cox regression coefficient.

\section{Validation}

$\mathrm{K}-\mathrm{M}$ survival analysis was performed on the prognostic genes in both training and validation groups, in order to assess the impact of a single hypoxia gene on the survival of HCC patients.

Firstly, all risk value of HCC patients in the training group was calculated according to the risk score formula. Then these patients were divided into high- and low-risk groups referring to the median score as a cutoff point. Secondly, K-M survival analysis was carried out to evaluate the predictive ability of the prognostic model. Thirdly, ROC curve is drawn to evaluate the sensitivity and specificity of the prognostic model for predicting survival outcomes. And the AUC would illustrate the accuracy of the prognosis. R package "survcomp" was applied to calculate the concordance index (C-index) of the model to further illustrate the predictive ability of the model. Similarly, the same performance was done in TCGA to evaluate the applicability of the model in an external database. All the above operations are implemented in $\mathrm{R}$ language.

\section{The clinical relevance of the model and production of the nomogram}

We performed univariate and multivariate cox regression analysis on the training group as well as the validation group to explore whether the hypoxia prognosis model is independent from other clinical factors. The "rms" software package is used in $\mathrm{R}$ language for the construction of the nomogram.

\section{GSEA}

GSEA was utilized to identify groups of related genes that were differentially expressed, further to investigate possible immune mechanisms associated with hypoxia [46]. The GSEA in this study was designed using h.all.v7.2.symbols.gmt. Normally, $P$ value $<0.05$ and FDR value $<0.05$ are considered to witness significant enrichment.

\section{Analysis on immune cell infiltration}

CIBERSORT is the tool most commonly used to analyze immune cell infiltration, which can assess the relative abundance of tumor infiltrating immune cells in different risk groups $[47,48]$. In this study, we used CIBERSORT to evaluate the proportion of 22 immune cells in all patients from ICGC and TCGA, and the sum of the scores of all estimated immune cell types is equal to 1 . And qualified samples were selected regarding to the standard of $P<0.05$. Simultaneously, box plots of highand low-risk groups were drafted in $\mathrm{R}$ language.

\section{Analysis on immune genes}

On the purpose of evaluating the expression of immune genes in the high- and low-risk hypoxia groups, we analyze the immunosuppressive genes in the ICGC and TCGA via $R$ language. A heat map of all genes was displayed, and related diagrams and box plots of key immune checkpoints were generated in $\mathrm{R}$ language.

\section{Abbreviations \\ HCC: Hepatocellular carcinoma; OS: Overall survival; TME: Tumor microenviron- ment; ICl: Immune checkpoint inhibitors; ICGC: International Cancer Genome Consortium; TCGA: The Cancer Genome Atlas; GSEA: Gene set enrichment analysis; PPI: Protein-protein interaction network; LASSO: Least Absolute Shrinkage and Selection operator; K-M: Kaplan-Meier; ROC: Receiver operat- ing characteristic; AUC: Area under curve; LCSGJ: Liver Cancer Study Group of Japan; AJCC: American Joint Committee on Cancer.}

\section{Supplementary Information}

The online version contains supplementary material available at https://doi. org/10.1186/s12876-021-02057-0.

Additional file 1. 200 hypoxia-related genes were acquired from the gene set enrichment analysis (GSEA) website.

Additional file 2. All immune-related genes downloaded from the Tracking Tumor Immuno-phenotype website.

\section{Acknowledgements}

Not applicable.

Authors' contributions

C.C. and L.Y. carried out the study, did analysis and interpretation of data. C.C. and K.Z. participated in paper design and drafted the manuscript together. All authors read and approved the final manuscript.

\section{Funding}

Not applicable.

\section{Availability of data and materials}

The datasets used and analyzed during the current study are available from the publicly open databases of ICGC (https://icgc.org/), TCGA (https://portal. gdc.cancer.gov) and GSEA (https://www.gsea-msigdb.org/).

\section{Declarations}

Ethics approval and consent to participate

Not applicable.

Consent for publication

Not applicable.

Competing interests

The authors declare that they have no competing interests.

\section{Author details}

${ }^{1}$ Emergency Department of Yinzhou No.2 Hospital, Ningbo 315000 , Zhejiang, China. ${ }^{2}$ Gastroenterology Department of Ningbo No. 9 Hospital, Ningbo 315000, Zhejiang, China.

Received: 18 August 2021 Accepted: 2 December 2021

Published online: 12 December 2021 


\section{References}

1. Sung H, Ferlay J, Siegel RL, Laversanne M, Soerjomataram I, Jemal A, Bray F. Global cancer statistics 2020: GLOBOCAN estimates of incidence and mortality worldwide for 36 cancers in 185 countries. CA Cancer J Clin. 2021:71(3):209-49.

2. Bray F, Ferlay J, Soerjomataram I, Siegel RL, Torre LA, Jemal A. Global cancer statistics 2018: GLOBOCAN estimates of incidence and mortality worldwide for 36 cancers in 185 countries. CA Cancer J Clin. 2018:68(6):394-424

3. Nault JC, Villanueva A. Biomarkers for hepatobiliary cancers. Hepatology (Baltimore, MD). 2021;73(Suppl 1):115-27.

4. Villanueva A. Hepatocellular carcinoma. N Engl J Med. 2019;380(15):1450-62.

5. Meurette $\mathrm{O}$, Mehlen P. Notch signaling in the tumor microenvironment. Cancer Cell. 2018;34(4):536-48.

6. Riera-Domingo C, Audigé A, Granja S, Cheng WC, Ho PC, Baltazar F, Stockmann C, Mazzone M. Immunity, hypoxia, and metabolism-the Ménage à Trois of cancer: implications for immunotherapy. Physiol Rev. 2020;100(1):1-102.

7. Jing X, Yang F, Shao C, Wei K, Xie M, Shen H, Shu Y. Role of hypoxia in cancer therapy by regulating the tumor microenvironment. Mol Cancer. 2019;18(1):157.

8. Fu C, An N, Liu J, Jun A, Zhang B, Liu M, Zhang Z, Fu L, Tian X, Wang D, et al. The transcription factor ZFHX3 is crucial for the angiogenic function of hypoxia-inducible factor $1 \mathrm{a}$ in liver cancer cells. J Biol Chem. 2020;295(20):7060-74.

9. Hu B, Yang XB, Sang XT. Development and verification of the hypoxiarelated and immune-associated prognosis signature for hepatocellular carcinoma. J Hepatocell Carcinoma. 2020;7:315-30.

10. Feng B, Zhu Y, Sun C, Su Z, Tang L, Li C, Zheng G. Basil polysaccharide inhibits hypoxia-induced hepatocellular carcinoma metastasis and progression through suppression of HIF-1a-mediated epithelial-mesenchymal transition. Int J Biol Macromol. 2019;137:32-44.

11. Yao B, Li Y, Chen T, Niu Y, Wang Y, Yang Y, Wei X, Liu Q, Tu K. Hypoxiainduced cofilin 1 promotes hepatocellular carcinoma progression by regulating the PLD1/AKT pathway. Clin Transl Med. 2021;11(3):e366.

12. Haber PK, Puigvehí M, Castet F, Lourdusamy V, Montal R, Tabrizian P, Buckstein M, Kim E, Villanueva A, Schwartz M, et al. Evidence-based management of HCC: systematic review and meta-analysis of randomized controlled trials (2002-2020). Gastroenterology. 2021;161(3):879-98.

13. Liu ZL, Liu JH, Staiculescu D, Chen J. Combination of molecularly targeted therapies and immune checkpoint inhibitors in the new era of unresectable hepatocellular carcinoma treatment. Ther Adv Med Oncol. 2021;13:17588359211018026.

14. Chen $Y$, Ramjiawan RR, Reiberger T, Ng MR, Hato T, Huang Y, Ochiai H, Kitahara S, Unan EC, Reddy TP, et al. CXCR4 inhibition in tumor microenvironment facilitates anti-programmed death receptor-1 immunotherapy in sorafenib-treated hepatocellular carcinoma in mice. Hepatology (Baltimore, MD). 2015:61(5):1591-602.

15. Su YY, Li CC, Lin YJ, Hsu C. Adjuvant versus neoadjuvant immunotherapy for hepatocellular carcinoma: clinical and immunologic perspectives. Semin Liver Dis. 2021;41(3):263-76.

16. Lindo L, Wilkinson LH, Hay KA. Befriending the hostile tumor microenvironment in CAR T-cell therapy. Front Immunol. 2020;11:618387.

17. Cheng J, Gao F, Chen X, Wu J, Xing C, Lv Z, Xu W, Xie Q, Wu L, Ye S, et al. Prohibitin-2 promotes hepatocellular carcinoma malignancy progression in hypoxia based on a label-free quantitative proteomics strategy. Mol Carcinog. 2014;53(10):820-32.

18. Jiang HY, Ning G, Wang YS, Lv WB. Ahypoxia-related signature enhances the prediction of the prognosis in hepatocellular carcinoma patients and correlates with sorafenib treatment response. Am J Transl Res. 2020:12(12):7762-81.

19. Luo X, Wei YQ, Hai L, Hu YC, Zhao ZJ, Ma WL, Ma LN, Liu XY, Ding XC. A preliminary study of serum marker alpha-enolase in the diagnosis of hepatocellular carcinoma. Chin J Hepatol. 2019;27(7):505-10.

20. Jiang K, Dong C, Yin Z, Li R, Mao J, Wang C, Zhang J, Gao Z, Liang R, Wang $\mathrm{Q}$, et al. Exosome-derived ENO1 regulates integrin a6 $\beta 4$ expression and promotes hepatocellular carcinoma growth and metastasis. Cell Death Dis. 2020;11(11):972

21. Hu Q, Shen S, Li J, Liu L, Liu X, Zhang Y, Zhou Y, Zhu W, Yu Y, Cui G. Low UGP2 expression is associated with tumour progression and predicts poor prognosis in hepatocellular carcinoma. Dis Markers. 2020;2020:3231273

22. Jiang H, Ma N, Shang Y, Zhou W, Chen T, Guan D, Li J, Wang J, Zhang E, Feng $Y$, et al. Triosephosphate isomerase 1 suppresses growth, migration and invasion of hepatocellular carcinoma cells. Biochem Biophys Res Commun. 2017;482(4):1048-53

23. Hamaguchi T, lizuka N, Tsunedomi R, Hamamoto Y, Miyamoto T, lida M, Tokuhisa Y, Sakamoto K, Takashima M, Tamesa T, et al. Glycolysis module activated by hypoxia-inducible factor 1alpha is related to the aggressive phenotype of hepatocellular carcinoma. Int J Oncol. 2008;33(4):725-31.

24. Bristow RG, Hill RP. Hypoxia and metabolism. Hypoxia, DNA repair and genetic instability. Nat Rev Cancer. 2008;8(3):180-92.

25. Yuan J, Narayanan L, Rockwell S, Glazer PM. Diminished DNA repair and elevated mutagenesis in mammalian cells exposed to hypoxia and low pH. Can Res. 2000;60(16):4372-6.

26. Kaplan AR, Glazer PM. Impact of hypoxia on DNA repair and genome integrity. Mutagenesis. 2020;35(1):61-8.

27. Yasuda S, Arii S, Mori A, Isobe N, Yang W, Oe H, Fujimoto A, Yonenaga Y, Sakashita H, Imamura M. Hexokinase II and VEGF expression in liver tumors: correlation with hypoxia-inducible factor 1 alpha and its significance. J Hepatol. 2004;40(1):117-23.

28. Chen R, Zhu S, Fan XG, Wang H, Lotze MT, Zeh HJ 3rd, Billiar TR, Kang R, Tang D. High mobility group protein B1 controls liver cancer initiation through yes-associated protein -dependent aerobic glycolysis. Hepatology (Baltimore, MD). 2018;67(5):1823-41.

29. Li X, Zhang K, Li Z. Unfolded protein response in cancer: the physician's perspective. J Hematol Oncol. 2011:4:8.

30. Bartoszewska S, Collawn JF. Unfolded protein response (UPR) integrated signaling networks determine cell fate during hypoxia. Cell Mol Biol Lett. 2020;25:18

31. Vandewynckel YP, Laukens D, Bogaerts E, Paridaens A, Van den Bussche A, Verhelst X, Van Steenkiste C, Descamps B, Vanhove C, Libbrecht L, et al. Modulation of the unfolded protein response impedes tumor cell adaptation to proteotoxic stress: a PERK for hepatocellular carcinoma therapy. Hepatol Int. 2015;9(1):93-104.

32. Sangro B, Sarobe P, Hervás-Stubbs S, Melero I. Advances in immunotherapy for hepatocellular carcinoma. Nat Rev Gastroenterol Hepatol. 2021:18(8):525-543.

33. Kim CG, Kim C, Yoon SE, Kim KH, Choi SJ, Kang B, Kim HR, Park SH, Shin EC Kim YY, et al. Hyperprogressive disease during PD-1 blockade in patients with advanced hepatocellular carcinoma. J Hepatol. 2021;74(2):350-9.

34. Donisi C, Puzzoni M, Ziranu P, Lai E, Mariani S, Saba G, Impera V, Dubois M, Persano M, Migliari M, et al. Immune checkpoint inhibitors in the treatment of HCC. Front Oncol. 2020;10:601240.

35. Liu F, Liu Y, Chen Z. Tim-3 expression and its role in hepatocellular carcinoma. J Hematol Oncol. 2018;11(1):126.

36. Noman MZ, Desantis G, Janji B, Hasmim M, Karray S, Dessen P, Bronte $V$, Chouaib S. PD-L1 is a novel direct target of HIF-1a, and its blockade under hypoxia enhanced MDSC-mediated T cell activation. J Exp Med. 2014;211(5):781-90

37. Barsoum IB, Smallwood CA, Siemens DR, Graham CH. A mechanism of hypoxia-mediated escape from adaptive immunity in cancer cells. Can Res. 2014;74(3):665-74.

38. Hou A, Hou K, Huang Q, Lei Y, Chen W. Targeting myeloid-derived suppressor cell, a promising strategy to overcome resistance to immune checkpoint inhibitors. Front Immunol. 2020;11:783.

39. Jiang M, Qin B, Luo L, Li X, Shi Y, Zhang J, Luo Z, Zhu C, Guan G, Du Y, et al. A clinically acceptable strategy for sensitizing anti-PD-1 treatment by hypoxia relief. J Control Release. 2021;335:408-19.

40. Ni JM, Ni AP. Landscape of PD-1/PD-L1 regulation and targeted immunotherapy. Chin Med Sci J. 2018;33(3):174-82.

41. Chen J, Wang X, Yuan Y, Chen H, Zhang L, Xiao H, Chen J, Zhao Y, Chang J, Guo W et al. Exploiting the acquired vulnerability of cisplatin-resistant tumors with a hypoxia-amplifying DNA repair-inhibiting (HYDRI) nanomedicine. Sci Adv. 2021;7(13):eabc5267.

42. Steingold JM, Hatfield SM. Targeting hypoxia-A2A adenosinergic immunosuppression of antitumor T cells during cancer immunotherapy. Front Immunol. 2020;11:570041.

43. Leone RD, Horton MR, Powell JD. Something in the air: hyperoxic conditioning of the tumor microenvironment for enhanced immunotherapy. Cancer Cell. 2015:27(4):435-6. 
44. Leone RD, Emens LA. Targeting adenosine for cancer immunotherapy. J Immunother Cancer. 2018;6(1):57.

45. Brown B, Weaver T, Wolfson J. MEBoost: Variable selection in the presence of measurement error. Stat Med. 2019;38(15):2705-18.

46. Subramanian A, Tamayo P, Mootha VK, Mukherjee S, Ebert BL, Gillette MA, Paulovich A, Pomeroy SL, Golub TR, Lander ES, et al. Gene set enrichment analysis: a knowledge-based approach for interpreting genome-wide expression profiles. Proc Natl Acad Sci USA. 2005;102(43):15545-50.

47. Newman AM, Steen CB, Liu CL, Gentles AJ, Chaudhuri AA, Scherer F, Khodadoust MS, Esfahani MS, Luca BA, Steiner D, et al. Determining cell type abundance and expression from bulk tissues with digital cytometry. Nat Biotechnol. 2019;37(7):773-82.

48. Newman AM, Liu CL, Green MR, Gentles AJ, Feng W, Xu Y, Hoang CD, Diehn M, Alizadeh AA. Robust enumeration of cell subsets from tissue expression profiles. Nat Methods. 2015;12(5):453-7.

\section{Publisher's Note}

Springer Nature remains neutral with regard to jurisdictional claims in published maps and institutional affiliations.

- fast, convenient online submission

- thorough peer review by experienced researchers in your field

- rapid publication on acceptance

- support for research data, including large and complex data types

- gold Open Access which fosters wider collaboration and increased citations

- maximum visibility for your research: over $100 \mathrm{M}$ website views per year

At BMC, research is always in progress.

Learn more biomedcentral.com/submissions 